\title{
On a High Dimensional Riemann's Mapping Theorem and Its Applications
}

\author{
Yukinobu Adachi ${ }^{1}$ \\ 1 2-12-29 Kurakuen, Nishinomiya, Hyogo, Japan \\ Correspondence: Yukinobu Adachi, 2-12-29 Kurakuen, Nishinomiya, Hyogo 662-0082, Japan. E-mail: \\ fwjh5864@nifty.com
}

Received: April 21, 2014 Accepted: June 3, 2014 Online Published: June 26, 2014

doi:10.5539/jmr.v6n3p13 URL: http://dx.doi.org/10.5539/jmr.v6n3p13

\begin{abstract}
We prove that the domain $D$ in $\Gamma \times \mathbf{C}_{z}$ where $\Gamma$ is a polydisk centered at $(0)$ and the fiber of $D$ over every point of $\Gamma$ is a simply connected domain in $\mathbf{C}_{z}$ which contains a small disk $\{|z| \leqq \varepsilon\}$, where $\varepsilon$ is independent of every point of $\Gamma$, is biholomorophic to some complete Hartogs domain. And we give applications of the uniformization of some fiber spaces.
\end{abstract}

Keywords: Riemann's mapping theorem, Robin-Yamaguchi function, complete Hartogs domain, uniformization of fiber spaces

\section{Introduction}

As for the classical uniformization problems such as the Hilbert's 22nd problem of a high dimensional case, there are some big theories of Griffith (1971) and Bers (1976). But the problem is not solved completely in the case of the manifold yet. For its special case such as an uniformization of a fiber space, Nishino (1969) proved that a Stein holomorphic family of $\mathbf{C}$-type covering Riemann surfaces over a complex plane over a disk is $\mathbf{C}^{1}$ bundle. Yamaguchi (1976) gave a simpler proof than that of Nishino and proved more simply with Maitani (2004) by another principle. In (Yamaguchi, 1976), he introduced a Robin-Yamguchi function $\lambda(t)$ which is the Robin constant of the Green function of a fiber $D_{t}$ when the base complex parameter of $t$ is fixed which has a pole at a point of $D_{t}$ which is independent to the parameter $t$, proved that $\log \lambda(t)$ is superharmonic under some conditions and solved an uniformization problem of a Stein holomorphic family of covering Riemann surfaces over $\mathbf{P}^{1}$ by using this fact in the case that the genus of them is zero and they are Riemann surfaces of parabolic type. His theorem includes the Nishino's result as a special case.

The fiber spaces studied by Nishino and Yamaguchi was the one over a disk. Fujita $(1979,1980,1987)$ extended their studies to fiber spaces over a polydisk and Yamaguchi and others also extended the Robin-Yamaguchi function of several complex variables and got many remarkable results especially in (Kim, Levenberg, \& Yamaguchi, 2011).

But the original problem of an uniformization of a Stein holomorphic family whose fibers are of hyperbolic type Riemann surfaces has been unsolved after that time except for a special case as in (Browder \& Yamaguchi, 1994).

In this note, we prove for a manifold $D$ such as a fiber space $D=(D, \pi, \Gamma)$ which is considered as a Riemann domain over $\Gamma \times \mathbf{P}^{1}$ where $\Gamma$ is a polydisk centered at (0), which has some schlicht branch and whose fibers over $\Gamma$ are simply connected ramified covering Riemann surfaces over $\mathbf{P}^{1}$ is mapped biholomorphically to a complete Hartogs domain (Theorem 5.1). This problem is reduced to a high dimensional Riemann's mapping theorem (Theorem 4.4).

We give some applications to the uniformization problem of fiber spaces which have topologicaly the same type fibers (Theorem 5.2 and 5.3).

Finally we have a local uniformization of a Stein holomorphic family whose fibers are topologically same type open Riemann surfaces (Definition 6.1) by reducing to a Riemann domain which can be applied to a high dimensional Riemann mapping theorem (Proposition 6.5 and Theorem 6.6). 


\section{Preliminary(1)}

Following proposition is well known.

Proposition 2.1 If there is a conformal map $\varphi$ from $\{|z|<r\}$ to $\{|w|<R\}$ with $\varphi(0)=0$ and $\varphi^{\prime}(0)=1$, then $r=R$ and $\varphi$ is an identity map for any $r$ with $0<r \leqq \infty$.

Corollary 2.2 Let $D$ be a simply connected domain in $\mathbf{C}_{z}$ such that $D \ni\{0\}$. Then the conformal map $\varphi$ of $D$ to $\{|w|<R\}$ with $\varphi(0)=0$ and $\varphi^{\prime}(0)=1$ is uniquely determined with $R(\leqq \infty)$.

Proposition 2.3 Let $D_{1}$ and $D_{2}$ be a simply connected domain in $\mathbf{C}_{z}$ such that $D_{2} \ni D_{1} \ni 0$ and $\varphi_{i}$ is a conformal map of $D_{i}$ to $\left\{|w|<R_{i}\right\}$ with $\varphi_{i}(0)=0$ and $\varphi_{i}^{\prime}(0)=1(i=1,2)$. Then $R_{1}<R_{2} \leqq \infty$.

Proof. If we consider the conformal map $\varphi_{2} \circ \varphi_{1}^{-1}$, we have $R_{1}<R_{2}$ by Schwarz's lemma when $R_{2}<\infty$ and we can easy to see when $R_{2}=\infty$ because $\varphi_{2}=i d$.

Proposition 2.4 Let $D$ be a bounded simply connected domain surrounded by a Jordan curve in $\mathbf{C}_{z}$ such as $0 \in D$. Let $\varphi$ be a conformal map of $D$ to $\{|w|<R\}$ with $\varphi(0)=0$ and $\varphi^{\prime}(0)=1$. Then $\log R$ is the Robin constant of the Green function of $D$ which has the pole at $z=0$ and $\varphi=R \cdot z \cdot \exp (-k(z))$ where $k(z)$ is some holomorphic function of $D$ with $k(0)=\log R$.

Proof. Above Green function $g=\log \left|\frac{R}{\varphi}\right|=-\log |z|+\log \left|\frac{z}{\varphi}\right| R$. When we set $u(z)=\log \left|\frac{z}{\varphi}\right| R, u(z)$ is a harmonic function and the Robin constant is $u(0)=\log R$.

Let $v(z)$ be a conjugate harmonic function of $u(z)$ with $v(0)=0$. When we set $k(z)=u(z)+i v(z), k(z)$ is holomorphic on $D$ and $k(0)=\log R$.

By virtue of Riemann's method, $w=\psi(z)=\exp [-\{g+i(-\arg z+v)\}]=\exp (\log |z|+\operatorname{iarg} z-k(z))=z \cdot \exp (-k(z))$ is a conformal map of $D$ to $|w|<1$. From Corollary $2.2, \varphi=R \cdot \psi$ since $R \cdot \psi(0)=0$ and $(R \cdot \psi)^{\prime}(0)=1$.

Proposition 2.5 Let $D$ be the same of Proposition 2.4. Let $f$ be a holomorphic function of $\bar{D}$ and $\lim _{z \rightarrow \partial D}|f(z)|=R$ with $f(0)=0, f(z) \neq 0$ for $z \neq 0$ and $f^{\prime}(0)=a \neq 0$. Then the Robin constant of the Green function $g$ of $D$ which has the pole at $z=0$ is $\log \frac{R}{|a|}$ and $f$ is a conformal map of $D$ to $\{|w|<R\}$.

Proof. Since $g=\log \left|\frac{R}{f}\right|=-\log |z|+\log \left|\frac{z}{f}\right| R$ is a Green function of $D$ which has the pole at $z=0$. It is easy to see that the Robin constant of $g$ is $\log \frac{R}{|a|}$ and $f$ is a conformal map of $D$ to $\{|w|<R\}$ by argument principle.

From Corollary 2.2 the following corollary is easy to see.

Corollary 2.6 If $a=1$ in the above proposition, $f=\varphi$ where $\varphi$ is the same of Proposition 2.4.

From Proposition 2.4, 2.5 and Corollary 2.6 the following proposition is easy to see.

Proposition 2.7 Let $D$ and $\varphi$ be the same of Proposition 1.4. Let $R_{0}$ is a positive constant such as $R_{0}<R$. If we set $D^{0}=\varphi^{-1}\left(|w|<R_{0}\right)$, then Robin constant of the Green function of $D^{0}$ which has the pole at $z=0$ is $\log R_{0}$.

\section{Preliminary(2)}

Definition 3.1 We denote a domain $H:=\left\{(t, w) ;|w|<R_{t} \leqq \infty\right.$ for $t \in \Delta(\rho)$ where $\Delta(\rho)$ is a disk centered at 0 with radius $\rho(0<\rho \leqq \infty)$ \}. We call $H$ a complete Hartogs domain. We denote by $H(R)$ if $R_{t} \equiv R$. (In the case (t) varies in a polydisk $\Gamma$ centered at $(0)$ we call it samely.)

Following proposition is well known essentially due to Hartogs.

Proposition 3.2 The complete Hartogs domain $H$ is a Stein one, if and only if $-\log R_{t}$ is subharmonic or identically $-\infty$ on $\Delta(\rho)$. (In the case $(t)$ varies in a polydisk $\Gamma, H$ is a Stein Hartogs domain if and only if $-\log R_{(t)}$ is plurisubharmonic or identically $-\infty$ on $\Gamma)$.

Proposition 3.3 Let $H$ be a complete Hartogs domain such that $-\log R_{t}$ be a function of $C^{2}$ class with $-\log R_{t}>$ $-\infty$. Then there is a biholomorphic map $\Phi$ of $H$ to $H(1)$ such as $\Phi(t, z)=(t, w), \Phi(t, 0)=(t, 0)$ if and only if $\log R_{t}$ is harmonic on $\Delta(\rho)$. (In the case $(t)$ varies in a polydisk $\Gamma$ and there is a biholomorphic map $\Phi$ of $H$, such as $-\log R_{(t)}$ is of $C^{2}$ class function with $-\log R_{(t)}>-\infty$, to $H(1)$ such as $\Phi((t), z)=((t), w), \Phi((t), 0)=((t), 0)$ if and only if $\log R_{(t)}$ is pluriharmonic on $\left.\Gamma\right)$.

Proof. When there is such a map $\Phi,|w|=\frac{|z|}{R_{t}}$ and $\frac{\partial^{2}}{\partial t \partial \hat{t}} \log |w|=\frac{\partial^{2}}{\partial t \partial \hat{t}} \log |z|-\frac{\partial^{2}}{\partial t \partial \hat{t}} \log R_{t}=0$ when $z \neq 0, \frac{\partial^{2}}{\partial t \partial \hat{t}} \log R_{t}=0$. When $\log R_{t}$ is harmonic, we set $\log R_{t}=u(t)$ where $t=x+i y, x, y \in \mathbf{R}$ and $t \in \Delta(\rho)$. And we set the 1 -form 
$\omega=-\frac{\partial u}{\partial y} d x+\frac{\partial u}{\partial x} d y$. Then $\omega$ satisfies the integrability condition because $u(t)$ is a harmonic function on $\Delta(\rho)$. Set $v(t)=\int_{0}^{x}-\frac{\partial u(s, 0)}{\partial y} d s+\int_{0}^{y} \frac{\partial u(x, r)}{\partial x} d r$. Then $u(t)$ and $v(t)$ satisfy Cauchy-Riemann differential equations. We set $f(t)=u(t)+i v(t), f(t)$ is holomorphic on $\Delta(\rho)$. Since $R_{t}=e^{u(t)}=\left|e^{u(t)+i v(t)}\right|=\left|e^{f(t)}\right|$, the map $\Psi: t=t, z=e^{f(t)} w$ is a biholomorphic one of $H(1)$ to $H$.

When the case $(t)$ varies in a polydisk $\Gamma$, refer to (Nishino, 2001, p. 14).

Corollary 3.4 There is no biholomorphic map $\Phi$ of $H_{T}:=\left\{(t, z) ; t \in \Delta(\rho),|z|<R_{t}=\left(R^{2}-|t|^{2}\right)^{\frac{1}{r}}, R>\rho>0, r>0\right\}$ (the Thullen type Stein domain) to $H(1)$ such that $\Phi(t, z)=(t, w)$ and $\Phi(t, 0)=(t, 0)$.

Proof. As $\frac{\partial^{2}}{\partial t \partial \bar{t}}\left(-\log R_{t}\right)=\frac{R^{2}}{r\left(R^{2}-|t|^{2}\right)^{2}}>0$, we have a conclusion from Proposition 3.3.

Remark 3.5 Let $H$ be the complete Hartogs domain and $R_{t}$ is continuous function of $t$. Then $H$ is homeomorphic to $H(R)$ with $0<R \leqq \infty$.

Definition 3.6 Let $D$ be a domain in $\Delta(\rho) \times \mathbf{C}_{z}$ and for every fixed $t_{0}$ in $\Delta(\rho), D_{t_{0}}:=D \cap\left\{t=t_{0}\right\}$ is a bounded simply connected domain surrounded by a $C^{2}$ smooth Jordan curve. And when $D_{t} \ni\{z=0\}$ for every $t \in \Delta(\rho)$ and $\bigcup_{t \in \Delta(\rho)} \partial D_{t}$ varies in $C^{2}$ class, that is, there is a defining real valued $C^{2}$ function in a neighborhood $\mathcal{V}$ of $\bigcup_{t \in \Delta(\rho)} \partial D_{t}$ in $\Delta(\rho) \times \mathbf{C}_{z}$ such as $\psi(t, z)$ such that $\bigcup_{t \in \Delta(\rho)} \partial D_{t}=\{\psi(t, z)=0\}$ which is a real submanifold of $C^{2}$ class in $\Delta(\rho) \times \mathbf{C}_{z}$, $D \cap \mathcal{V}=\{\psi(t, z)<0\}$ and $\mathcal{V} \cap \bigcup_{t \in \Delta(\rho)}\left(\overline{D_{t}}\right)^{c}=\{\psi(t, z)>0\}$, we call such $D$ a domain of class (A).

We set

$$
k_{2}(t, z)=\left(\frac{\partial^{2} \psi}{\partial t \partial \bar{t}}\left|\frac{\partial \psi}{\partial z}\right|^{2}-2 \operatorname{Re}\left\{\frac{\partial^{2} \psi}{\partial \bar{t} \partial z} \frac{\partial \psi}{\partial t} \frac{\partial \psi}{\partial \bar{z}}\right\}+\left|\frac{\partial \psi}{\partial t}\right|^{2} \frac{\partial^{2} \psi}{\partial z \partial \bar{z}}\right) /\left|\frac{\partial \psi}{\partial z}\right|^{3} .
$$

We remark that it does not depend on the choice of defining function $\psi(t, z)$ of $\bigcup_{t \in \Delta(\rho)} \partial D_{t}=\{\psi(t, z)=0\}$ with the property of the above definition.

Proposition 3.7 (cf. Shiffer (1946, pp. 417, 418) and also Theorem 3.1 in Maitani and Yamaguchi (2004)) Let $D \in(A)$. Then the Green function of $D_{t}$ which has the pole at $z=0$ (especially the Robin constant $\log R_{t}$ of $\left.D_{t}\right)$ varies in $C^{2}$ class with respect to the variable $t$.

Lemma 3.8 (Theorem 3.1 in Maitani and Yamaguchi (2004)) Let $D \in(A)$ and $\Phi$ be a map of $D$ to $H$ such that $\Phi$ : $t=t, w=\varphi(t, z)$ where $\varphi(t, z)$ is a conformal map of $D_{t}$ to $\left\{|w|<R_{t}\right\}$ such that $\varphi(t, 0)=0$ and $\frac{\partial \varphi}{\partial z}(t, 0)=1$.

Then, for the Robin-Yamaguchi function $\log R_{t}$ of $D$ and for the Green function $\log \frac{R_{t}}{|\varphi|},-\log \frac{R_{t}}{|\varphi|}$ is extended to a defining function of $\bigcup_{t \in \Delta(\rho)} \partial D_{t}$ such as $D \in(A)$ and the following equality is concluded.

$\frac{\partial^{2}}{\partial t \partial \bar{t}} \log R_{t}=-\frac{1}{\pi} \int_{\partial D_{t}} k_{2}(t, z)\left|\frac{\partial}{\partial z} \log \frac{R_{t}}{|\varphi|}\right|^{2} d s-\frac{4}{\pi} \iint_{D_{t}}\left|\frac{\partial^{2}}{\partial t \partial \bar{z}} \log \frac{R_{t}}{|\varphi|}\right|^{2} d u d v$

where $\partial D_{t}=\{z(s)\}, z=u+i v$, ds is the arc length element of $\partial D_{t}$ and $k_{2}$ is above definition which means the Levi-curvature of $\bigcup_{t \in \Delta(\rho)} \partial D_{t}$.

Corollary 3.9 Let the situation be above and D be a Stein domain. Then

$\frac{\partial^{2}}{\partial t \partial \hat{t}} \log R_{t} \leqq-\frac{4}{\pi} \iint_{D_{t}}\left|\frac{\partial^{2}}{\partial t \partial \bar{z}} \log \frac{R_{t}}{|\varphi|}\right|^{2} d u d v \leqq 0, \log R_{t}$ is a superharmonic function on $D$ and $H$ is a complete Hartogs Stein domain.

Proof. The first statement is easy to see from Lemma 3.8 and $k_{2} \geqq 0$ because $D$ is a Stein domain. The second one is a well known fact when the first statement is true. The third one is followed by Proposition 3.2.

Following proposition is owed essentially to H. Yamaguchi.

Proposition 3.10 Let $D$ be a Stein domain, $D \in(A)$ and the Robin-Yamaguchi function $\log R_{t}$ be harmonic on $\Delta(\rho)$. Then $D$ is Levi-flat $\left(k_{2} \equiv 0\right)$ and $\Phi$ is biholomorphic map from $D$ to $H$ where $\Phi$ is the same in Lemma 2.8.

Proof. By Lemma 3.8 and the assumption such as $\frac{\partial^{2}}{\partial t \partial \bar{t}} \log R_{t}=0$ and $k_{2} \geqq 0, k_{2} \equiv 0$ and $\left|\frac{\partial^{2}}{\partial t \partial \bar{z}} \log \frac{R_{t}}{|\varphi|}\right|=0$ when $z \neq 0$. As $\log \frac{R_{t}}{|\varphi|}$ is a real valued function, $\frac{\partial^{2}}{\partial \bar{t} \partial z} \log \frac{R_{t}}{|\varphi|}=0$ when $z \neq 0$. Then $\frac{\partial^{2}}{\partial \bar{t} \partial z} \log |\varphi|=0$ when $z \neq 0$. Since $\frac{\partial}{\partial z} \log |\varphi|=\frac{1}{2} \frac{\frac{\partial \varphi}{\partial z}}{\varphi}$ and $\varphi=R_{t} \cdot z \cdot \exp (-k(t, z))$ by Proposition $1.4, \frac{\partial}{\partial t}\left\{\frac{1}{2}\left(\frac{1}{z}-\frac{\partial}{\partial z} k(t, z)\right)\right\}=0$ and $\frac{\partial^{2}}{\partial \bar{t} \partial z} k(t, z)=0$. Since $k(t, 0)=\log R_{t}$ by Proposition 2.4, k(t,z)- $\log R_{t}=\int_{0}^{z} \frac{\partial}{\partial z} k(t, z) d z$ and $\frac{\partial}{\partial \bar{t}}\left(k(t, z)-\log R_{t}\right)=\int_{0}^{z} \frac{\partial^{2}}{\partial \bar{t} \partial z} k(t, z) d z=0$.

Since $\frac{\partial \varphi}{\partial \bar{t}}=z \cdot \exp (-k(t, z)) R_{t}\left\{\frac{\partial}{\partial \bar{t}}\left(\log R_{t}-k(t, z)\right)\right\}=0$, the function $\varphi=R_{t} \cdot z \cdot \exp (-k(t, z))$ is holomorphic function of variables $t$ and $z$ separately and it is holomorphic by Hartogs theorem. Then $\Phi$ is biholomorphic.

Theorem 3.11 Let $D \in(A)$. Then $D$ is a Stein domain and the Robin-Yamaguchi function $\log R_{t}$ is harmonic on 
$\Delta(\rho)$ if and only if there is a biholomorphic map $\Phi_{0}$ of $D$ to $H(1)$ such that $\Phi_{0}(t, 0)=(t, 0), \Phi_{0}(t, z)=(t, w)$ and $D$ is Levi-flat.

Proof. When $D$ is a Stein domain and $\log R_{t}$ is harmonic on $\Delta(\rho), D$ is Levi-flat and $\Phi: D \rightarrow H$ is a biholomorphic map by Proposition 3.10 where $\Phi$ is the same in Lemma 3.8 and there is a map $\Phi_{0}$ as above by Proposition 3.3.

When there is a map $\Phi_{0}$ as above, $D$ is a Stein domain because $H(1)$ is a Stein domain. Set $\Phi_{0}$ with $\Phi_{0}: t=t, w=$ $\varphi_{0}(t, z)$ where $\varphi_{0}(t, 0)=0, \frac{\partial}{\partial z} \varphi_{0}(t, 0) \neq 0$. Then $\varphi_{0}(t, z)=a_{1}(t) z+a_{2}(t) z^{2}+\cdots$, where $a_{1}(t) \neq 0$ locally near $\{z=0\}$. By Proposition 2.5, $\log R_{t}=-\log \left|a_{1}(t)\right|$ and it is harmonic on $\Delta(\rho)$ because of $a_{1}(t) \neq 0$ and holomorphic. Then $D$ is Levi-flat by Proposition 3.10.

\section{Conclusion}

Lemma 4.1 Let $D$ be a domain of in $\Delta(\rho) \times \mathbf{C}_{z}$ such that when an arbitrary number $t_{0}$ in $\Delta(\rho), D_{t_{0}}=D \cap\left\{t=t_{0}\right\}$ is a simply connected domain and $D_{t_{0}} \supset\{|z| \leqq \varepsilon\}$ where $\varepsilon$ is a positive constant which is independent of $t_{0}$. We asumme that $\Phi$ is the map of $D$ to $H$ such as $t=t, w=\varphi(t, z)$, which is a conformal map of $D_{t}$ to $\left\{|w|<R_{t} \leqq \infty\right\}$ such as $\varphi(t, 0)=0$ and $\frac{\partial}{\partial z} \varphi(t, 0)=1$ for fixed $t$. Then there is a constant $\delta$ such that a domain $H(\delta)$ with $\delta<R_{t}$ for every $t \in \Delta(\rho)$ and $\Phi^{-1}(H(\delta))=D^{0}$ is a domain of the class $(A)$ and $\varphi(t, z)$ is holomorphic on $D^{0}$.

Proof. There is a small constant $\delta$ above by the modified Koebe one-quarter theorem. From Proposition 2.7 the Green function $g_{0}$ of $D^{0}$ which has the pole at $z=0$ is $\log \frac{\delta}{|\varphi|}$. From Proposition $3.7-g_{0}=\log \frac{|\varphi|}{\delta}$ is a defining function of $D^{0}$ if $\mathcal{V}=D^{\prime}=\Phi^{-1}\left(H\left(\delta^{\prime}\right)\right)$ where $\delta$ and $\delta^{\prime}$ are nealy equal and $\delta<\delta^{\prime}$ and $D^{0} \in(A)$ of Definition 3.6.

$\operatorname{Set} \psi=\log \frac{|\varphi|}{\delta}$ and $\varphi(t, z)=\delta \cdot z \cdot \exp (-k(t, z))$ anew. Since $\psi$ is a real valued function, $\frac{\partial \psi}{\partial t}=\frac{1}{2} \frac{\frac{\partial \varphi}{\partial t}}{\varphi}=-\frac{1}{2} \frac{\partial k}{\partial t}, \frac{\partial \psi}{\partial \bar{t}}=-\frac{1}{2} \frac{\partial \bar{k}}{\partial \bar{t}}$ and $\frac{\partial^{2} \psi}{\partial \bar{t} \partial t}=-\frac{1}{2} \frac{\partial^{2} k}{\partial \bar{t} \partial t}=-\frac{1}{2} \frac{\partial^{2} \vec{k}}{\partial \bar{t} \partial t}$. We set $k(t, z)=u(t,(x, y))+i v(t,(x, y))$ where $z=x+i y$. Then $v(t,(x, y))$ is a harmonic function of a variable $t$ since $\frac{\partial^{2}}{\partial \bar{t} \partial t}(k-\bar{k})=\frac{\partial^{2}}{\partial \bar{t} \partial t}(2 i v(t,(x, y)))=0$ by above equation. And $u(t,(x, y))$ is a harmonic one also because

$$
\frac{\partial^{2}}{\partial t \partial \bar{t}} u(t,(x, y))=\int_{0}^{x}-\frac{\partial}{\partial y}\left(\frac{\partial^{2}}{\partial t \partial \bar{t}} v(t,(s, 0))\right) d s+\int_{0}^{y} \frac{\partial}{\partial x}\left(\frac{\partial^{2}}{\partial t \partial \bar{t}} v(t,(x, r))\right) d r=0 .
$$

As $u(t,(x, y)), v(t,(x, y))$ are harmonic functions of a variable $t, \frac{\partial^{2} k}{\partial t \partial t}=\frac{\partial}{\partial t}\left(\frac{\partial k}{\partial t}\right)=0$ and $\frac{\partial k}{\partial t}$ is a holomorphic function of a variable $t$. Therefore $k(t, z)$ is a holomorphic one of $t$. By Hartogs theorem $k$ is a holomorphic function of $(t, z)$ because it is holomorphic with respect to $t$ and $z$ separately. Then $\varphi(t, z)$ is a holomorphic function on $D^{0}$.

Theorem 4.2 In the same situation of above lemma, the map $\Phi$ is biholomorphic.

Proof. Since $\varphi(t, z)$ is holomorphic on $D^{0}$ and holomorphic of a variable $z$ when $t$ is fixed in $\Delta(\rho), \varphi(t, z)$ is a holomorphic function on $D$ by virtue of Hartogs theorem.

From Theorem 4.2, Proposition 3.2 and 3.3, the following corollary is easy to see.

Corollary 4.3 Let $D$ be the same of Lemma 4.1. Then $D$ is a Stein domain if and only if $-\log R_{t}$ is subharmonic or identically $-\infty$ on $\Delta(\rho)$. And $D$ is biholomorphic to $H(1)$ if and only if $-\log R_{t}$ is harmonic on $\Delta(\rho)$.

Theorem 4.4 Let $D$ be a domain in $\Gamma \times \mathbf{C}_{z}$ where $\Gamma:=\left\{\left(t_{1}, \ldots, t_{n}\right) ;\left|t_{i}\right|<\rho_{i}, 0<\rho_{i} \leqq \infty, i=1, \ldots, n\right\}$ and for every point $\left(t_{0}\right) \in \Gamma, D_{\left(t_{0}\right)}:=D \cap\left\{(t)=\left(t_{0}\right)\right\}$ is a simply connected domain and $D_{\left(t_{0}\right)} \supset\{|z| \leqq \varepsilon\}$ for every $\left(t_{0}\right) \in \Gamma$ where $\varepsilon$ is a positive number which is independent of $\left(t_{0}\right)$. Let $\log R_{(t)}$ be the Robin-Yamaguchi function of $D_{(t)}$.

Then the map $\Phi$ of $D$ to a complete Hartogs domain $H$ such as $(t)=(t), w=\varphi((t), z)$, which is a conformal map of $D_{(t)}$ to $\left\{|w|<R_{(t)} \leqq \infty\right\}$ with fixed $(t)$ such as $\varphi((t), 0)=0$ and $\frac{\partial}{\partial z} \varphi((t), 0)=1$, is biholomorphic.

Proof. If we fix $\left(t_{1}, \ldots, t_{n}\right)$ except $t_{i}(1 \leqq i \leqq n)$ and fix $z \in\{|z| \leqq \varepsilon\}, \varphi$ is holomorphic with respect to $t_{i}$ by Theorem 4.2. If we fix $(t), \varphi$ is holomorphic with respect to $z$. Then $\varphi$ is holomorphic on $\Gamma \times \mathbf{C}_{z}$ by Hartogs theorem. It is easy to see that $\Phi$ is biholomorphic.

From Theorem 4.4, Proposition 3.2 and 3.3, the following corollary is easy to see.

Corollary 4.5 In the same situation of Theorem 4.4, D is a Stein domain if and only if $-\log R_{(t)}$ is plurisubharmonic on $\Gamma$ or identically $-\infty$. And $D$ is biholomorphic to $H(1)$ if and only if $-\log R_{(t)}$ is pluriharmonic on $\Gamma$.

\section{Applications}

Following theorem is easy to see by the same method of Theorem 4.4 and the uniformization theorem by Koebe.

Theorem 5.1 Let $D=(D, \pi, \Gamma)$ be a connected manifold such as a ramified Riemann domain over $\Gamma \times \mathbf{P}^{1}$ with 
the projection $\pi$ where $\Gamma$ is the same of Theorem 4.4 and it has a schlicht branch $D^{0}$ over $\Gamma \times\{|z| \leqq \varepsilon\}$ for some $\varepsilon>0$. And we assume that for every $(t) \in \Gamma$, the fiber $D_{(t)}$ is an irreducible ramified covering Riemann surface such as simply connected. Then $D$ is biholomorphic to some complete Hartogs domain $H$ in $\Gamma \times\{|w| \leqq \infty\}$ with fiber preserving.

Theorem 5.2 Let $D$ is the same of Theorem 5.1. We assume that $D$ is a Stein manifold and for every $(t) \in \Gamma$, the fiber $D_{(t)}$ is an irreducible ramified covering Riemann surface with genus $g(\leqq \infty)$ and the number of boundary components is $n(1 \leqq n \leqq \infty)$ where $g$ and $n$ are independent of $(t)$. We call such $D$ of $(g, n)$ type.

Then we have the following results (I) and (II):

(I) If $(g, n)=(0,1), D_{(t)}$ is simply connected and there are following cases:

(a) When for every $(t) \in E$ which is a capacity positive set with $E \subset \Gamma, D_{(t)}$ is holomorphically isomorphic to $\mathbf{C}, X$ is biholomorphic to $H(\infty)$.

(b) When for every $(t) \in \Gamma$ except for a capacity zero set, $D_{(t)}$ is holomorphically isomorphic to the unit disk, $D$ is biholomorphic to a complete Hartogs Stein domain $H$ (The definition of the set of capacity zero or positive, see Fujita, 1987, p. 685).

$\left(b^{\prime}\right)$ When for every $(t) \in \Gamma, D_{(t)}$ is holomorphically isomorphic to the unit disk, $D$ is biholomorphic to $H$ with $R_{t}<\infty$ and $D^{\prime}=\left(\pi^{-1}\left(\Gamma^{\prime}\right), \pi, \Gamma^{\prime}\right)$ where $\Gamma^{\prime}$ is the arbitrary polydisk centered at $(0)$ such as $\Gamma^{\prime} \Subset \Gamma$ is biholomorphic to some bounded complete Hartogs Stein domain $H^{\prime}$.

(II) If $(g, n) \neq(0,1)$ and $X$ is homeomorphic to $\Gamma \times R$ where $R$ is a Riemann surface of $(g, n)$ type, the universal covering space $\widetilde{D}$ of $D$ with base point $((t), o) \in D^{0}$ where o is the point in $D_{t}^{0}$ whose projection is $z=0, \widetilde{D}$ is homeomorphic to $\Gamma \times \widetilde{R}$ and there are following cases:

(c) When $\widetilde{D_{(t)}}$ is the case $(a)$ of $(I)$, that is $D_{(t)}$ is holomorphically isomorphic to $\mathbf{C}^{*}$ for a capacity positive set $E \subset \Gamma, \widetilde{D}$ is biholomorphic to $H(\infty)$.

(d) When $\widetilde{D_{(t)}}$ is the case $(b)$ of $(I), \widetilde{D}$ is biholomorphic to $H$ as the same of $(b)$ of $(I)$.

$\left(d^{\prime}\right)$ When $\widetilde{D_{(t)}}$ is the case $\left(b^{\prime}\right)$ of $(I), \widetilde{D}$ is biholomorphic to $H$ with $R_{t}<\infty$ and $\widetilde{D^{\prime}}$ is biholomorphic to some bounded complete Hartogs Stein domain $H^{\prime}$ which is samely defined as above $\left(b^{\prime}\right)$ of $(I)$.

Proof. In the case (I), we define a map $\Phi$ of $D$ to $H$ where $\Phi((t), \varphi((t), p))=((t), w)$, where $w=\varphi((t), p)$ is a conformal map of $D_{(t)}$ to $|w|<R_{(t)}(\leqq \infty)$ with $\varphi(t, o)=0, \frac{\partial}{\partial z} \varphi((t), o)=1$ by the uniformization theorem by Koebe where $p$ is a point of $D_{(t)}$. Then the function $\varphi((t), p)$ is uniquely determined.

By Theorem 4.4, $\varphi((t), p)$ is holomorphic on $D^{0}$. By Hartogs theorem, $(t, \varphi)$ is biholomorphic from $D$ to $H$. Since $H$ is a complete Hartogs Stein domain of $\Gamma \times \mathbf{C},-\log R_{(t)}$ is plurisubharmonic or identically $-\infty$ by Proposition 3.2. When $-\log R_{(t)}=-\infty$ for a capacity positive set $E \subset \Gamma,-\log R_{(t)} \equiv-\infty$ on $\Gamma$ by the nature of plurisubharmonic function and the case (a) occurs. When $-\log R_{(t)} \not \equiv-\infty,-\log R_{(t)}>-\infty$ except for a capacity zero set of $\Gamma$ and the case (b) occurs. When $-\log R_{(t)}>-\infty$ on $\Gamma,-\log R_{(t)} \leqq M$ on $\Gamma^{\prime}$ where $M$ is a real constant and the case $\left(b^{\prime}\right)$ occurs.

The case (c), (d) and ( $\left.d^{\prime}\right)$ of (II) is easy to see from the above discussion because $\widetilde{D}$ is a Stein manifold and biholomorphic to a complete Hartogs Stein domain.

Theorem 5.3 Let $D=(D, \pi, \Gamma)$ be a manifold such as a ramified Riemann domain over $\Gamma \times \mathbf{P}^{1}$ and for every $(t) \in \Gamma$, the fiber $D_{(t)}$ is a compact Riemann surface with genus $g(\geqq 1)$ which is independent of $(t)$ where $\Gamma$ is the same of Theorem 4.4. And it has a schlicht branch $D^{0}$ such as over $\Gamma \times\{|z| \leqq \varepsilon, \varepsilon>0\}$.

Then $\widetilde{D}$ which is constructed as the same way of Theorem 5.2 (II), is biholomorphic to $\Gamma \times \mathbf{C}$ when $g=1$ and $\widetilde{D}$ is biholomorphic to a complete Hartogs domain $H$ when $g \geqq 2$ where the Hartogs radious $R_{(t)}<\infty$ for every $(t) \in \Gamma$.

Proof. When $g=1, \widetilde{D}$ is in the case (I) (a) of Theorem 5.2 and when $g \geqq 2, \widetilde{D_{(t)}}$ is holomorphically isomorphic to the unit disk, above statement is easy to see from Theorem 5.1.

\section{Reduction}

Definition 6.1 (cf. Definition 3.4 in Adachi, 2001) Let $D$ be a Stein manifold of dimension $n+1(n \geqq 1)$, $\Gamma:=$ $\left\{\left(t_{1}, \cdots, t_{n}\right) ;\left|t_{i}\right|<\rho_{i}, 0<\rho_{i} \leqq \infty, i=1, \cdots, n\right\}$ and $\pi:=\left(f_{1}, \cdots, f_{n}\right)$ be a holomorphic map of $D$ onto $\Gamma$ such that

(1) for any $(t) \in \Gamma, \pi^{-1}(t)=D_{(t)}$ is an one dimensional irreducible analytic subset of $D$, 
(2) for the set $\{p \in D$; the rank of Jacobian of $\pi$ at $p<n\}$ is contained in a hypersurface $S$ of $D$ and $S \cap D_{(t)}$ are isolated points in $D_{(t)}$ for every $(t) \in \Gamma$

and

(3) $(D, \pi, \Gamma)$ is homeomorphic to $\Gamma \times R$ preserving fibers where $R$ is an open Riemann surface.

Then we call $D=(D, \pi, \Gamma)$ a Stein holomorphic family of open Riemann surfaces with respect to $R$.

Proposition 6.2 Let $D$ be the same above. Then the canonical bundle $K_{D}$ has a glovbal holomorphic section $g$ which has zero only on $S$ with any high order. If $S=\emptyset$, we take $g$ such as $g \neq 0$.

Proof. Let $\left\{\varphi_{i j} \in O^{*}\left(U_{i} \cap U_{j}\right), U_{i}, U_{j} \subset D\right\}$ be the transition function of $K_{D}$. Then there are solutions of the Cousin II problem such that $\left\{\varphi_{i} \in O\left(U_{i}\right)\right\}$ since $D$ is a Cousin II domain by Oka principle. As $\left\{\varphi_{i} \in O\left(U_{i}\right)\right\}$ is a Cousin II data, there is a holomorphic function $h$ on $D$ such that $\left\{\frac{\varphi_{i}}{h}, U_{i}\right\}$ is a non-zero holomorphic section of $K_{D}$. Let $k$ be a holomorphic function on $D$ whose zero sets are $S$. Since $D$ is a Cousin II domain, there is always such a $k$. Let $m$ be an arbitrary positive integer.Then $\left\{\frac{k^{m}}{h} \varphi_{i}, U_{i}\right\}=\left\{g_{i}, U_{i}\right\}$ is a holomorphic section $g$ of $K_{D}$ which satisfies the condition above.

Proposition 6.3 Let $D=(D, \pi, \Gamma)$ be a Stein holomorphic family of open Riemann surfaces with respect to $R$ which is simply connected. Then it is biholomorphic to a brached Riemann domain over $\Gamma \times \mathbf{C}$. When $S=\emptyset$, it is biholomorphic to an unbranched Riemann domain over $\Gamma \times \mathbf{C}$.

Proof. We consider the linear differential equation $\frac{\partial\left(f_{1}, \cdots, f_{n}, u\right)}{\partial\left(v_{\alpha}^{1}, \cdots, v_{\alpha}^{n+1}\right)}=g$ (1) where $g$ is the same of Proposition 6.2, $\left(v_{\alpha}^{1}, \cdots, v_{\alpha}^{n+1}\right)$ are any coordinate variables of $D$ and $u$ is an unknown function.

By virtue of the Cauchy-Kovalevskaya theorem, there is a neighborhood $W$ of a point $p$ in $D-S$ where $\pi(p)$ is an arbitrary point of $\Gamma$ and a local holomorphic solution $u_{W}$ in $W$ of (1) where $\left.u_{W}\right|_{\sigma}=0$ where $\sigma$ is a local holomorphic section of $D$ through $p$. By the same method of Lemma 2.2 in (Adachi, 2001) the equation (1) can be regarded as an analytic family of holomorphic 1-forms on fibers $D_{(t)}=\left\{f_{1}=t_{1}, \cdots, f_{n}=t_{n}\right\}$ which can be considered as characteristic curves of (1) because we can take $g$ which has zero only on $S$ with sufficient high order if the integer $m$ in the proof of above proposition take sufficient larage. The local solution $u_{W}$ has an analytic continuation along each fiber endlessly through $W$ and $u_{W}$ is single valued holomorphic function on $\left(\pi^{-1}(W), \pi, \pi(W)\right)$ and a global holomorphic solution $u \in O(D)$ from Lemma 2.2 in (Adachi, 2001).

Set $F=\left(f_{1}, \ldots, f_{n}, u\right)$. Then it is a holomorphic map from $D$ to $\Gamma \times \mathbf{C}$. It is easy to see from the condition (2) of Definition 5.1 that $F$ is scatterd inverse map. Then from Osgood theorem, $F^{-1}$ defines a branched Riemann domain over $\Gamma \times \mathbf{C}$ which is biholomorphic to $D$. When $S=\emptyset$, it is biholomorphic to an unbranched Riemann domain over $\Gamma \times \mathbf{C}$ because we can take $g$ such as $g \neq 0$ from Proposition 6.2.

Proposition 6.4 If $R$ is not simply connected, the universal covering Stein manifold $\tilde{D}=(\tilde{D}, \tilde{\pi}, \Gamma)$ which is homeomorphic to $\Gamma \times \tilde{R}$ is biholomorphic to a branched Riemann domain over $\Gamma \times \mathbf{C}$. When $S=\emptyset, \tilde{D}$ is biholomorphic to an unbranched Riemann domain over $\Gamma \times \mathbf{C}$.

Proof. We consider the eqation (1) in the proof of Proposition 6.3. We use the same notations in it. The local holomorphic solution $u_{W}$ in $W$ of (1) where $\left.u_{W}\right|_{\sigma}=0$ has an analytic continuation along any path on each fiber. Since the universal covering space $\tilde{D}$ is constructed by takeing the universal covering of every fiber, $F$ is a holomorphic map from $\tilde{D}$ to $\Gamma \times \mathbf{C}$ because $\tilde{D}$ is a Stein manifold and $F^{-1}$ defines a branched Riemann domain over $\Gamma \times \mathbf{C}$ which is biholomorphic to $\tilde{D}$. When $S=\emptyset$, it is easy to see the conclusion by the same reason of the proof of above proposition and above discussion.

Proposition 6.5 Let $D=(D, \pi, \Gamma)$ be a Stein hoomorphic family of open Riemann surfaces with respect to $R$ which is an arbitrary Riemann surface. When it has a global holomorphic section $\sigma$ with $\sigma \cap S=\emptyset$, for every polydisk $\Gamma^{\prime}$ centered $(0)$ with $\Gamma^{\prime} \Subset \Gamma \widetilde{D^{\prime}}=\left(\widetilde{\pi^{-1}\left(\Gamma^{\prime}\right)}, \pi, \Gamma^{\prime}\right)$ has a schlicht branch $\left(D^{0}\right)^{\prime}$ over $\Gamma^{\prime} \times\{|z| \leqq \varepsilon\}$ for some $\varepsilon>0$. When $S=\emptyset$, we have above conclusion always easily if we take $\Gamma^{\prime}$ suficiently small.

Proof. Since the holomorphic map $F: \tilde{D} \rightarrow \Gamma \times \mathbf{C}$ in the proof of above proposition such as $\left.F\right|_{\sigma}=0$ and Jacobian of $F$ is not 0 near $\Gamma^{\prime} \times\{0\}$, it is easy to see the conclusion above when we take $\Gamma^{\prime}$ small again if necessaly.

Theorem 6.6 Let $D=(D, \pi, \Gamma)$ be a Stein holomorphic family of open Riemann surfaces with respect to an arbitrary Riemann surface $R$. For every $(t) \in \Gamma$ there is a neighborhood $v(t) \Subset \Gamma$ such that $\tilde{D^{\prime}}=\left(\widetilde{\pi^{-1}(v)}, \pi^{\prime}, v\right)$ is biholomorphic to to some Hartogs Stein domain $H^{\prime}$ in $\gamma \times \mathbf{C}$ where $\gamma$ is a polydisk centered at (0) which is biholomorphic to $v(t)$. 
Proof. From Proposition 6.5 and Theorem 5.1 it is easy to see the above conclusion.

\section{Appendix}

Problem 7.1 Let $T=\left(P_{1}\left(x_{1}, \cdots, x_{n+1}\right), \cdots, P_{n+1}\left(x_{1}, \cdots x_{n+1}\right)\right): \mathbf{C}^{n+1} \rightarrow \mathbf{C}^{n+1}\left(\xi_{1}, \cdots, \xi_{n+1}\right)$ be a polynomial map with $J T \equiv 1$. When the set $\left\{\xi_{1}=P_{1}, \cdots, \xi_{n}=P_{n}\right\}$ is not empty and an irreducible curve for every $(\xi) \in \mathbf{C}^{n}$, is $T \in \operatorname{Aut}\left(\mathbf{C}^{n+1}\right)$ ?

Remark 7.2 In the case $n=1$, Kaliman in 1993 proved that $\left\{\xi_{1}=P_{1}\left(x_{1}, x_{2}\right)\right\}$ is irreducible for every $\xi_{1} \in \mathbf{C}$ when $J T \equiv 1$. And we proved in (Adachi, 2011) that $\left\{\xi_{1}=P_{1}\left(x_{1}, x_{2}\right)\right\}$ is simply connected for every $\xi_{1} \in \mathbf{C}$ by reducing to the Theorem 2.1 in (Adachi, 2001). Then it is easy to see that $T \in \operatorname{Aut}\left(\mathbf{C}^{2}\right)$ if $J T \equiv 1$ (Theorem 3.2 in Adachi, 2011, that is a proof of two dimensonal Jacobian conjecture).

Remark 7.3 Even in the case $n=1$, there is a $P\left(x_{1}, x_{2}\right)$ where $\left\{\xi=P\left(x_{1}, x_{2}\right)\right\}$ is irreducible for every $\xi \in \mathbf{C}$ and $\{\xi=P\}$ is not simply connected. See for it's example in (Essen, 2000, p. 256).

Proposition 7.4 We assume further that for every $(\xi) \in \mathbf{C}^{n}$, the fiber $\{(\xi)=(P)\}$ is an irreducible algebraic curve of the same type $(g, n)$, then $T \in A u t\left(\mathbf{C}^{n+1}\right)$.

Proof. Let $D=\mathbf{C}^{n+1}=\left(\mathbf{C}^{n+1},(P), \mathbf{C}^{n}\right)$ and $R$ be an algebraic Riemann surface of $(g, n)$ type. Then $D$ is a Stein holomorphic family with respect to $R$. Then $\tilde{D}=\mathbf{C}^{n+1}$ is a Stein holomorphic family with respect to $\tilde{R}$. Then if we consider the following linear partial differencial equation on $\tilde{D}$ :

$$
\frac{\partial\left(P_{1}, \cdots, P_{n}, u\right)}{\partial\left(x_{1}, \cdots, x_{n+1}\right)}=g
$$

has a global solution $u$ for an arbitrary $g \in O\left(\mathbf{C}^{n+1}\right)$ from Theorem 2.1 in (Adachi, 2001) in $\tilde{D}$ and $u$ is a single valued holomorphic function on $\mathbf{C}^{n+1}$. From Theorem 3.2 in (Adachi (2001)), $T \in A u t\left(\mathbf{C}^{n+1}\right)$.

Problem 7.5 In the same notation of Problem 7.1, is the set $\left\{\xi_{1}=P_{1}, \cdots, \xi_{n}=P_{n}\right\}$ not empty and an irreducible curve of the topologicall same type for every $(\xi) \in \mathbf{C}^{n}$ when $J T \equiv 1$ ?

\section{References}

Adachi, Y. (2001). Condition for global exsistence of holomorphic solutions of a certain differential equation on a Stein domain of $\mathbf{C}^{n+1}$ and its applications. J. Math. Soc. Japan, 53, 633-644. http://dx.doi.org/10.2969/jmsj/1213023727

Adachi, Y. (2011). On the Jacobian conjecture. Pioneer J. Math. and Math. Sci., 2, 101-111.

Bers, L. (1976). On Hilbert's 22nd problem. Proc. Symposia in Pure Math., 28, 559-609. http://dx.doi.org/10.1090/pspum/028.2/9903

Browder, A., \& Yamaguchi, H. (1994). A variation formula for harmonic modules and its application to several complex variables. Hiroshima Math. J., 24, 493-520.

Essen, A. (2000). Polynomial automorphisms and the Jacobian conjecture. Progress in Mathmatics, 190. Verlag Basel: Birkhäuser.

Fujita, O. (1979). Sur les systèmes de fonctions holomorphes de plusieurs variables complexes. J. Math. Kyoto Univ., 19, 231-254.

Fujita, O. (1980). Sur les systèmes de fonctions holomorphes de plusieurs variables complexes (II) Points critiques des applications. J. Math. Kyoto Univ., 20, 417-433.

Fujita, O. (1987). Sur les systèmes de fonctions holomorphes de plusieurs variables complexes (III) Uniformité des fibres d'une application. J. Math. Kyoto Univ., 27, 679-708.

Griffith, P. (1971). Complex-analytic properties of certain Zariski open sets on algebraic varieties. Ann. Math., 94, 21-51. http://dx.doi.org/10.2307/1970733

Kaliman, S. (1993). On the Jacobian conjecture. Proc. Amer. Math. Soc. 117, 45-91. http://dx.doi.org/10.1090/S0002-9939-1993-1106179-7

Kim, K-T., Levenberg, N., \& Yamaguchi, Y. (2011). Robin functions for complex manifolds and applications. Memories of A.M.S, 209.

Maitani, F., \& Yamaguchi, H. (2004). Variation of Bergman metrics on Riemann surfaces. Math. Ann., 330, 
477-489. http://dx.doi.org/10.1007/s00208-004-0556-8

Nishino, T. (1969). Nouvelles recherches sur les fonctions entières de plusieurs variables complexes (II). Fonction entières qui se réduisent à celles d'une variables. J. Math. Kyoto Univ., 9, 221-274.

Nishino, T. (2001). Function Theory in Several Complex Variables. Math. Monographs of the A.M.S., 193.

Shiffer, M. (1946). Hadamard's formula and variation of domain-functions. Amer. J. Math., 68, $417-448$. http://dx.doi.org/10.2307/2371824

Yamaguchi, H. (1976a). Parabolicité d'une fonction entière. J. Math. Kyoto Univ., 16, 71-92.

Yamaguchi, H. (1976b). Famille holomorph de surfaces de Riemann ouverts, qui est une variété de Stein. J. Math. Kyoto Univ., 16, 71-92.

\section{Copyrights}

Copyright for this article is retained by the author(s), with first publication rights granted to the journal.

This is an open-access article distributed under the terms and conditions of the Creative Commons Attribution license (http://creativecommons.org/licenses/by/3.0/). 